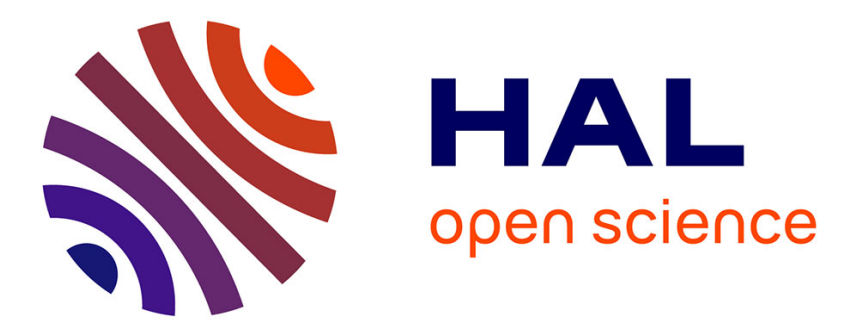

\title{
Les débuts de la physique mathématique et théorique au Brésil et l'influence de la tradition française.
}

\author{
Michel Paty
}

\section{To cite this version:}

Michel Paty. Les débuts de la physique mathématique et théorique au Brésil et l'influence de la tradition française.. Petitjean, Patrick; Jami, Catherine; Moulin, Anne-Marie;. Science and Empire, historical studies about scientific development and european expansion., Kluwer, pp.173-191, 1992. halshs-00167107

\section{HAL Id: halshs-00167107 https://shs.hal.science/halshs-00167107}

Submitted on 16 Aug 2007

HAL is a multi-disciplinary open access archive for the deposit and dissemination of scientific research documents, whether they are published or not. The documents may come from teaching and research institutions in France or abroad, or from public or private research centers.
L'archive ouverte pluridisciplinaire HAL, est destinée au dépôt et à la diffusion de documents scientifiques de niveau recherche, publiés ou non, émanant des établissements d'enseignement et de recherche français ou étrangers, des laboratoires publics ou privés. 
in Petitjean, Patrick; Jami, Catherine and Moulin, Anne-Marie (eds.), Science and Empire, historical studies about scientific development and european expansion, Kluwer, Dordrecht, 1992, p. 173-191.

\section{Les débuts de la physique mathématique et théorique au Brésil et l'influence de la tradition française}

\section{Introduction.}

La formation, tardive, d'une véritable communauté scientifique au Brésil s'est effectuée selon des modalités très différentes d'une discipline à une autre. Le développement de la physique théorique a été plus long à se réaliser que celui d'autres secteurs de la connaissance scientifique, et peut être daté pour l'essentiel des années trente de ce siècle, bien que l'on compte quelques noms importants et des contributions non négligeables dans les périodes antérieures. Il est intéressant de s'interroger sur les divers facteurs qui ont influé sur cette discipline et sur les circonstances qui ont précédé et préparé son émergence, aboutissant à déterminer une voie propre et originale, une véritable 'tradition' (ou école) nationale. Ces facteurs tiennent, considérant la spécificité de ce champ scientifique, pour une part au contexte local, aux structures mentales et institutionnelles, pour une autre aux influences culturelles et intellectuelles. Si l'on peut caractériser de façon relativement aisée ces deux groupes de facteurs conjoncturels, il est plus difficile d'évaluer exactement la part de chacun d'eux et les effets de leurs inter-relations. L'approche historique tend souvent, par la nature même de ses objets et de ses méthodes, à dissocier le contexte, qu'il est difficile de ne pas ramener à ses seuls aspects sociaux ou 
culturels au sens large, et le contenu, considéré sous des formes 'standardisées', en raison de l'objectivité de la science à laquelle on le rapporte. Le présent travail voudrait contribuer, à partir d'un cas d'espèce et bien délimité, à éclairer cette question délicate en essayant d'échapper à cette réduction.

Les circonstances de la formation d'une 'science brésilienne', tant au niveau d'une organisation systématique de l'enseignement supérieur dans toutes les disciplines qu'à celui d'une contribution significative à la recherche, ont été étudiées par divers auteurs ${ }^{1}$, qui ont insisté à juste titre sur les aspects institutionnels, et montré comment c'est la fondation, relativement récente, des Universités (et notamment celle de São Paulo en 1934) qui a donné l'impulsion déterminante. Les structures de formation et les quelques institutions de recherche qui existaient auparavant avaient été conçues dans un but pragmatique. L'enseignement supérieur se réduisait à des écoles techniques (Ecoles de Droit, de Médecine, des Mines², d'ingénierie, ces dernières au début militaires ${ }^{3}$ ), dont la création remonte pour l'essentiel à la fin du dix-neuvième siècle, et qui visaient à former des spécialistes directement utilisables pour répondre aux besoins de la société (ceux du Brésil lui-même, et aussi sans doute ceux des Puissances intéressées à certaines activités $\left.{ }^{4}\right)$. Quant à la recherche institutionalisée, elle visait également l'utilité, et était cantonnée à certains domaines: les historiens font généralement débuter l'institutionalisation de la science au Brésil avec la fondation de l'Institut sérothérapique de Manguinhos, à Rio, en 1900, voué à la recherche microbiologique et parasitologique, parce que son rôle et sa renommée furent considérables. Le retard au développement scientifique peut se mesurer par la distance qui sépare les années 1880-1890, où a lieu la création des premiers instituts techniques, et $1934^{5}$, date de la fondation de la première université

\footnotetext{
${ }^{1}$ Cf. Azevedo [1955], Ferri, Motoyama [1979-1981, Schwartzmann [1979].

2 L'école des Mines d'Ouropreto fut fondée en 1875 ; elle eût pour premier directeur Henri Gorceix (1842-1920), appelé de France par l'empereur don Pedro II.

3 L'Académie Militaire Royale, qui fut longtemps le seul lieu d'enseignement supérieur des mathématiques et des sciences physiques, fut fondée du temps de la Couronne portugaise, en 1810. Elle s'appela plus tard Ecole Militaire, et donna lieu ultérieurement à l'Ecole Centrale (1858) et à l'Ecole Polytechnique de Rio (1874).

4 Par exemple, et ceci vaut pour l'enseignement comme pour la recherche, la connaissance des maladies tropicales, ou celle des ressources minières et leur exploitation.

5 Cf. p. ex. Mathias [1982].
} 
moderne (l'USP, Université de l'Etat de São Paulo, bientôt suivie d'autres Universités fédérales et d'Etats).

C'est dans les années trente que se constitue, en même temps que d'autres disciplines, et à la faveur de la création et du développement de l'Université, la physique théorique brésilienne. Elle bénéficie, certes, de l'appui indispensable de l'Institution; et il n'est pas indifférent que ce soit sous l'impulsion de Teodoro Ramos, mathématicien et physicien théoricien qui occupe une position charnière, que la physique, théorique aussi bien qu'expérimentale (et plus généralement les sciences exactes) aît été développée à l'Université de São Paulo ${ }^{6}$. Dans cette réalisation, on ne saurait surestimer le soutien et le rôle actif de savants étrangers de grande envergure, fixés au Brésil pendant des années ${ }^{7}$, tel Gleb Wataghin (venu du laboratoire de Fermi en Italie) pour la physique. Mais si la 'greffe' a pris, de telle sorte que la physique (ainsi que d'autres branches, comme les mathématiques, la chimie, la biologie, pour s'en tenir aux sciences exactes ou naturelles), s'est développée d'une manière assez remarquable, par l'apparition de scientifiques de premier plan et de recherches originales du meilleur niveau, dès la fin des années trente et jusqu'aujourd'hui, le 'terrain' n'est évidemment pas indifférent: certaines conditions indispensables étaient présentes. C'est, précisément, sur ces conditions locales, que nous pouvons dire 'de préparation' d'un terrain favorable, mais peut-être en même temps porteuses de certaines limitations, que nous voudrions porter notre attention dans ce qui suit. Nous l'examinerons sous l'angle de l'interaction entre des éléments de connaissance, empruntés pour une grande part à une tradition-mère, et des facteurs contingents qui tiennent aux circonstances locales, donnant lieu à une assimilation originale que nous essaierons de caractériser.

Nous insisterons, dans ce travail, sur la période préliminaire, avant que ne commence de s'affirmer un courant à proprement parler de physique mathématique et théorique: cette période comprend le dixneuvième siècle et les premières années du vingtième ${ }^{8}$, se concluant, en

6 Sur la constitution de l'Université de São Paulo, et le recrutement de professeurs européens en sciences exactes (par Teodoro Ramos) et en sciences humaines (par Georges Dumas), cf., p. ex., Petitjean [1988, 1991].

7 Notamment les années de guerre. La science brésilienne a bénéficié de l'émigration européenne de savants fuyant le nazisme, mais à un degré bien moindre que les EtatsUnis.

8 Ce travail s'inscrit dans une recherche (cf. Paty, Petitjean [1985]), qui porte également sur les physico-mathématiciens brésiliens du premier tiers du vingtième siècle (Paty 
un certain sens, avec la création, à Rio de Janeiro en 1916, de la Société Brésilienne des Sciences, qui devint plus tard l'Académie. L'exhaustivité est ici bien entendu impossible, et nous nous en tiendrons à l'examen de quelques uns des traits les plus significatifs pour notre propos.

[1987, et en prép. 1]) et sur la réception de la théorie de la Relativité au Brésil (Paty [en prép. 2]). 


\section{Constitution d'une tradition scientifique et influences.}

\section{Facteurs institutionnels et idéologiques.}

La constitution progressive d'une science brésilienne fait partie d'un ensemble de modifications survenues dans le pays depuis la moitié du dix-neuvième siècle. Le Brésil colonial se transforme peu à peu, socialement et culturellement, et cette période est celle d'un essai d'adaptation progressive des structures de la société brésilienne aux exigences d'une nation moderne, promise à l'autonomie depuis l'Indépendance (1822), préoccupée de définir et d'affirmer son identité, ouverte au développement agricole, industriel et urbain et au peuplement de son espace (d'ailleurs sur l'arrière-fond d'une immigration massive). Des institutions se créent et une idéologie se forme, qui mènera à celle affirmée par la République, en 1889. Mais cette société est marquée en même temps par la persistance de structures archaïques, et même féodales, et l'avènement de sa République sera en vérité surtout une victoire de l'oligarchie. Son passé de colonie vouée à l'enrichissement de la Métropole la marquera longtemps (l'absence d'Université en est un exemple, lié d'ailleurs à la spécificité de la colonisation par le Portugal). D'autre part, s'il s'est détaché économiquement et politiquement du Portugal, le Brésil dépend depuis lors - et pratiquement jusqu'à la seconde guerre mondiale -, de l'Angleterre du point de vue économique. Quand à la 'dépendance culturelle', elle est plus complexe, autant et peut-être davantage choisie qu'imposée, mais elle n'en est pas moins présente, car les élites sociales brésiliennes garderont longtemps les yeux tournés vers l'Europe. Parmi les pays européens, c'est à la France que va très largement la préférence, pour des raisons qui ne sont pas propres au Brésil seul, puisque c'est le cas de la plupart des autres pays d'Amérique latine, voire d'autres pays dans le monde qui s'ouvrent à la modernité, et ces raisons tiennent à l'histoire autant qu'à la culture?

Dans ce contexte se situe l'influence du positivisme de Comte. Cette philosophie correspondait à des conceptions et à une attitude qui présentaient cet intérêt, pour les élites sociales brésiliennes, d'être attentives aux idées de progrès et, en même temps,

9 L'image de la France des Lumières tient un rôle considérable dans cet éveil à la modernité, et la Révolution n'en est pas séparée, qui assure la rupture avec l'ordre ancien - et qui d'ailleurs eût une influence sur les mouvements d'indépendance des pays latinoaméricains au début du dix-neuvième siècle. Voir, en particulier, Coggiola [1990], Petitjean [1989], ainsi que Dantes [1987]. 
fondamentalement conservatrices ${ }^{10}$. Ce n'est pas notre propos ici d'analyser les modifications subies par la doctrine de Comte dans son adaptation au contexte intellectuel et social brésilien ${ }^{11}$ : nous nous contenterons d'indiquer que la philosophie positiviste a pu stimuler, pour ce qui concerne notre sujet, la création d'Ecoles supérieures et d'Instituts de recherche appliquée, voire susciter un intérêt pour les sciences physiques et naturelles. Mais la "synthèse subjective" du Maître, reprise par ses disciples en France et au Brésil12, en proclamant que la connaissance est unilatéralement ordonnée à l'homme, dépréciait en même temps son aspect objectif. Elle constituait un handicap face au caractère fondamental de la connaissance scientifique et de la recherche, et même un blocage à l'égard de bien des conceptions modernes. Cependant, cet aspect d'une influence culturelle d'ordre général, dont il faut voir à quel niveau exactement elle s'exerce, ne doit pas masquer d'autres facteurs, qui pourront être éventuellement plus importants. Nous y reviendrons.

\footnotetext{
10 On sait que la devise de la République brésilienne, qui figure sur le drapeau national, est celle-là même d'Auguste Comte, "Ordem e progresso", "Ordre et progrès".

11 Cf., p. ex., Arantes [1988].

12 Cf. Comte [1856] et, p. ex., Lafitte [1875]. Sur l'histoire du positivisme au Brésil, voir Lins [1964], Arbousse-Bastide [1956 ].
} 


\section{Influences culturelles et scientifiques.}

La question des influences scientifiques soulève le problème de l'évaluation de l'état de développement de la science dans les centres ou métropoles, notamment dans le cas de la France, qui fut la source étrangère principale d'influence culturelle et scientifique au Brésil dans la période considérée. Les historiens qui se sont intéressés à l'histoire sociale des sciences ont parfois opposé l'ancienne splendeur de la période classique, c'est-à-dire le dix-septième et le dix-huitième siècles, où la culture et la science française excellaient, et le dix-neuvième qui, passé le premier tiers du siècle serait, avec le vingtième, le temps de sa décadence $^{13}$. Dans ces conditions, la considération privilégiée encore répandue de par le monde au début du vingtième pour cette culture et surtout pour cette science serait totalement injustifiée, celle-ci ne devant son reste de réputation qu'au prestige de son passé ; et l'on fait éventuellement valoir que la persistance de cette influence aurait été franchement négative, lui attribuant bien des facteurs du retard dans les pays qui l'on subie. Il pourrait être tentant, dans ce sens, d'imputer à l'excès d'influence française les retards de la science brésilienne, du moins en ce qui concerne la physique, invoquant un effet du positivisme en matière de sciences théoriques, ou la situation dans laquelle se trouvait, en France, au tournant du siècle, la physique théorique, alors qu'apparaissaient deux directions de nouveautés qui allaient la modifier radicalement, la Relativité, la Physique des Quanta, auxquelles elle serait restée largement étrangère.

Mais ce serait évidemment simplifier à l'extrème les situations en les schématisant, et ce serait surtout les déformer. En premier lieu en raison du caractère excessif de la thèse du déclin, qui résiste difficilement à l'examen. Sans exclure l'effet de facteurs institutionnels importants, comme l'organisation de la science française et son lien aux structures de l'Etat - en particulier à travers les Grandes Ecoles - qui a pu porter préjudice au dynamisme de la recherche, il s'agit là de tendances, non d'un état de chose de portée absolue. D'autres auteurs ont fait valoir, avec plus de vraisemblance, le caractère relatif de la prépondérance, à partir du moment où d'autres centres se sont développés, comme, au cours du dix-neuvième siècle, les pays de langue allemande et, plus généralement, d'Europe du Nord. Sans être le déclin de la science française, c'était du moins la fin d'une certaine hégémonie. Le grand physicien autrichien Ludwig Boltzmann témoignait en 1905,

13 Voir, p. ex., Ben David [1970], [Gilpin 1968]. 
dans son Voyage d'un professeur allemand en Eldorado ${ }^{14}$, de la concurrence entre les grands centres scientifiques européens de l'époque - et par là-même de leur qualité - en se plaignant de ce que, dans les congrès scientifiques qui se tenaient en Amérique (du Nord, en l'occurrence), "parmi les foreigners (les non-anglais) c'étaient toujours et partout les Français qui étaient les meilleurs". Et il regrettait l'absentéisme de ses collègues de l'Académie de Berlin, en s'écriant : "Nous, les Allemands, n'avons aucune raison de ne pas les égaler".

En second lieu, un schéma généralisant d'explication $a$ priori, d'ailleurs idéologique, s'avère insufisant en face de la réalité historique qui se présente d'abord de manière différentielle, et pour laquelle on ne saurait faire l'économie d'une étude spécifique, pour chaque domaine et chaque discipline, des différents facteurs qui marquent tant les traditions-mères dont l'influence se fait sentir, que les traditions locales sur lesquelles cette influence s'exerce. Un tel examen sera propre à faire voir les mécanismes exacts de constitution de traditions et du rôle des influences. Ces traditions privilégient de fait certains domaines, certains types d'approches et de problèmes, sans qu'il soit possible de les réduire à un jugement de valeur global. Par exemple, d'être demeurées délibérément à l'écart de certaines orientations, comme l'intérêt pour les fondements et l'axiomatisation, n'a pas empêché les mathématiques françaises au tournant du siècle d'être d'une qualité incontestable. De même, si la physique théorique proprement dite trouve peu de praticiens en France à la fin du dix-neuvième, la physique mathématique et la physique expérimentale n'ont pas cessé d'y connaître un riche développement.

Comme c'est précisément cette particularité disciplinaire que nous voudrions éclairer dans le cas de la science brésilienne aussi, disons en quelques mots quelle est cette distinction.

\section{Physique mathématique, physique théorique.}

La physique mathématique et la physique théorique, confondues jusqu'alors sous le signe de la mécanique rationnelle, et qui le demeurent pour l'astronomie mathématique (de Laplace à Poincaré), se séparent au début du dix-neuvième siècle avec la théorisation de domaines de la physique pour lesquels les principes et les concepts fondamentaux doivent faire l'objet d'une élaboration particulière. Les noms de Laplace, Fourier, Poisson évoquent bien ce qu'est la physique

14 Boltzmann [1905]. 
mathématique, par leur approche analytique (d'ailleurs féconde) des phénomènes de l'optique physique, de l'électricité, de la mécanique des fluides ou de la chaleur. L'utilisation du formalisme mathématique l'emporte sur l'élaboration physique proprement dite, laquelle est souvent ramenée à la seule considération d'un modèle général, tel le modèle moléculaire, permettant l'interprétation du formalisme.

La physique théorique, au contraire, met l'accent sur les caractères spécifiques des phénomènes considérés et formule des énoncés de nature physique avant toute mathématisation, énoncés qui d'ailleurs conditionnent cette dernière, comme on le voit par exemple dans les travaux de Fresnel en Optique, d'Ampère (puis de Faraday et Maxwell) en Electrodynamique, plus tard de Maxwell, Clausius, Boltzmann, en Thermodynamique (sur la base des deux principes de cette science), puis de Planck et d'Einstein.

Si la physique mathématique s'est instituée en une tradition riche et vivace en France, on ne peut dire qu'il en soit allé de même avec la physique théorique, dont les initiateurs n'ont pas eu d'héritiers directs dans leur pays, et qui fleurit très vite au contraire en Grande Bretagne et en Europe du Nord, sur les traces mêmes de Fresnel et Ampère. A vrai dire, la physique théorique qui se développa dans ces régions fut également héritière des Lagrange, Laplace, Poisson, Fourier, comme en témoignent ses représentants que furent, notamment, von Helmholtz, Kirchhoff, Hertz, Boltzmann, Lorentz... Il faudra attendre, pour qu'elle se voie cultivée de façon systématique en France, les années vingt du présent siècle, à l'occasion du développement de la théorie de la Relativité puis de la physique quantique. 


\section{Les premiers éléments constitutifs au dix-neuvième siècle.}

\section{Les étapes de la constitution de la physique comme discipline au Brésil.}

On rencontre, au Brésil, une situation un peu semblable, toutes proportions gardées puisqu'il s'agit d'une communauté scientifique naissante, et avec un certain décalage. Pour l'étudier à travers les scientifiques qui se sont intéressés à la physique (qu'elle soit mathématique, théorique ou expérimentale), il convient de distinguer diverses phases dans la constitution de la physique comme discipline au Brésil. La première est celle des tout premiers commencements, et comprend le dix-neuvième siècle et les premières années du vingtième : elle est marquée par quelques individualités remarquables, du mathématicien Joaquim Gomes de Souza à l'ingénieur et astronome Oto de Alencar (sans oublier Henrique Morizé pour la recherche expérimentale), et par le contexte d'un enseignement scientifique et technique qui connaît une forte influence du positivisme. C'est de cette période que nous esquisserons dans ce qui suit l'analyse : on y verra comment une situation locale se forme en empruntant des éléments dans deux directions fort diverses à une même source d'influence, qu'elle transforme selon des modalités déterminées par les particularités du contexte local.

La seconde phase, directement issue de la première en continuité comme en opposition, est celle d'une tradition en voie de constitution, qui s'affirme dans la direction de la physique mathématique mais s'ouvrant à la physique théorique proprement dite. Cette époque est marquée par des scientifiques de qualité, comme Amoroso Costa, Teodoro Ramos, Luis Freire et d'autres, qui se révèlent et font école, modifient les structures de l'institution, modernisent autant qu'ils le peuvent le contenu des enseignements ${ }^{15}$. Il est utile de remarquer que ce courant de physique mathématique, à travers lequel la physique théorique s'imposera peu à peu, est très influencé par la tradition des physico-mathématiciens français, de Henri Poincaré à Paul Painlevé, Emile Picard et Emile Borel. La troisième phase commence avec la création des Universités et voit toute une génération de jeunes chercheurs, formés par les précédents, développer une physique théorique, une physique mathématique et une physique expérimentale,

15 Pour une étude de leurs 'profils' dans le prolongement du présent travail, voir Paty [en prép. 1]. 
tant dans un enseignement systématique que dans des recherches originales qui rejoignent le concert international, et contribuent à faire de la science brésilienne l'une des plus avancées des nations neuves. Ces développements, qui se font à travers une diversification, voire un changement des influences, restent cependant tributaires des premiers inspirateurs.

Ayant ainsi situé les grandes lignes de l'évolution de la physique au Brésil, revenons à la première phase, dont les 'lignes de champ' marqueront les développements ultérieurs.

\section{Deux directions d'accès aux mathématiques}

L'histoire de l'implantation des mathématiques et de la physique au Brésil porte dès son origine la marque d'une influence française privilégiée. Sur l'ancienneté de cette influence, on évoquera tout d'abord le fait que les Universités d'Europe où des brésiliens allaient de préférence se former au temps de la colonie étaient non seulement celle de Coimbra, mais aussi celles de Montpellier et de Paris ${ }^{16}$. Ensuite, la pénétration dans le pays des mathématiques françaises, par des ouvrages dans l'édition originale ou traduits, dès 1800 , donc avant même l'arrivée de João VI à l'occasion de l'invasion du Portugal par les troupes napoléoniennes - arrivée qui, paradoxalement, eût pour effet d'amplifier l'influence culturelle en général de la France. Les premières traductions en portugais de Lazare Carnot ou de Lagrange à parvenir au Brésil, furent publiées à Lisbonne en 1798, mais sont l'oeuvre d'un brésilien : elles suivirent de près les parutions à Paris ${ }^{17}$. Puis c'est au Brésil même que les traductions s'éffectuèrent, à partir de 1809 , concernant surtout des livres de caractère didactique, ceux entre autres de Legendre et de Lacroix, qu'allait adopter l'Académie Militaire Royale ${ }^{18}$.

Nous savons que les mathématiques et la physique théorique étaient alors très étroitement liées ; mais c'est vers cette époque qu'elles se différencient dans la pratique des savants, et cette différence, prolongée dans celle entre la physique mathématique et la physique théorique, permet de comprendre un caractère très spécifique des débuts

16 Azevedo 1947, vol. 1.

17 Il s'agit des Réflexions sur la métaphysique du calcul infinitésimal (1797) de L. Carnot et de la Théorie des fonctions analytiques (1797) de Lagrange, traduites par Manuel Jacinto Nogueira da Gama.

18 L'Académie Militaire Royale, fondée en 1910, avait au programme l'étude des oeuvres de Euler, Bezout, Monge, Legendre, Lacroix, Laplace, Prony, Delambre, Lacaille, Delandre, Haüy, Brisson, etc. (Oliveira Castro [1955]). 
de la tradition brésilienne en physique. Tout au long du dix-neuvième siècle, les mathématiques et la physique seront cultivées au Brésil par une petite élite formée dans les Ecoles techniques, mais ne seront pas l'objet de contributions originales, à une exception près, d'ailleurs assez considérable pour qu'on s'y arrête. Nous reviendrons plus loin sur certains traits de cette 'assimilation' à partir des ouvrages importés d'Europe, qui a fourni, en tout état de cause, les moyens de dépasser les limitations qui tenaient à l'enseignement figé des Ecoles.

Deux éléments d'influence, tous deux originaires de France, ont en effet marqué, dans des directions en grande partie contraires, l'accès aux mathématiques et à la physique. Le premier vient d'être mentionné : il concerne la 'réception' des connaissances dans ces disciplines, à partir des publications disponibles, et la figure de Joaquim Gomes de Souza, bien qu'elle sorte du commun, constitue un exemple significatif, révélateur des dimensions de cette assimilation qui peut aller jusqu'à la création. Le second est celui d'une adaptation locale particulière du positivisme, dont les effets ont marqué de manière importante la formation d'enseignants et de techniciens au long de plusieurs décennies. 


\section{Le "premier physico-mathématicien brésilien".}

Joaquim Gomes de Souza, que l'on a pu qualifier de "premier physico-mathématicien brésilien"19, et que sa mort précoce (à l'âge de 34 ans) empêcha, dit-on, de réaliser une oeuvre plus considérable, donna en mathématiques et en physique mathématique des contributions originales. Cet autodidacte de génie, qui prépara seul et très jeune encore les examens de mathématiques et de physique de l'Ecole Militaire, était au fait des connaissances les plus récentes dans ce domaine. Sa 'thèse' d'astronomie théorique, soutenue à 19 ans et qui lui valut d'enseigner dans cette Ecole, réflétait la découverte, survenue deux ans auparavant, de la planète Neptune prédite par Le Verrier : le jeune savant posait le problème de savoir si, connaissant la perturbation d'une planète, il est possible de décider par l'analyse s'il existe plus d'une solution, c'est-à-dire une ou plusieurs planètes perturbatrices ${ }^{20}$. Il le résolvait dans la lignée des méthodes laplaciennes en mécanique céleste.

Ses contributions originales portent sur le calcul intégral et sur des applications à des problèmes de physique : théorie du son, détermination des fonctions inconnues d'intégrale définie, méthodes générales d'intégration considérant les conditions aux limites auxquelles doivent être soumises les solutions, énoncés et démonstrations de théorèmes d'analyse mathématique. Ces travaux, qui témoignent d'une connaissance approfondie de l'oeuvre des grands mathématiciens et physico-mathématiciens Euler, Lagrange, Laplace, Liouville, Abel, et manifestent "une grande intuition et un admirable talent d'analyste"21, firent l'objet de communications à l'Académie des Sciences de Paris en 1855. Le physicien anglais Stokes en estima certains résultats suffisamment importants pour qu'il les présentât devant la Royal Society de Londres, l'année suivante 22 .

Plus tard, son 'disciple' Oto de Alencar prendra certains de ces travaux pour base des siens propres, marquant la continuité de la physique mathématique brésilienne en voie de constitution. Que

19 Costa Ribeiro [1955], p. 167. Sur Joaquim Gomes de Souza (1829-1863), voir également Amoroso Costa [1929], p. 75-76, Freire [1931], Henry [1882], Oliveira Castro [1955], p. 56-61, Ramos [1929 b].

20 Gomes de Souza [1848]. Voir Morais [1955].

21 Ramos [1929 b]

22 Ces mémoires furent réunis avec d'autres dans une publication posthume, préfacée par le français Charles Henry, à l'initiative du gouvernement brésilien: Mélanges de Calcul intégral, Leipzig, 1882 (Gomes de Souza [1882]). 
l'Académie de Paris n'aît pas jugé bon de les publier est peut-être dû à ce qui pouvait alors apparaître comme manque de rigueur, mais qui était en fait la marque d'un esprit plus intuitif et créateur que formaliste : Gomes de Souza n'hésitait pas à envisager l'utilisation des séries divergentes, qu'il regardait comme "une espèce de symbole qui remplace la fonction génératrice", et sur lequel on peut effectuer des opérations, anticipant par là sur des idées qui s'avèreraient fécondes ${ }^{23}$.

Outre l'analyse, il s'intéressa aux équations algébriques, voyant dans ces dernières une analogie avec les équations différentielles linéaires et formulant l'espoir qu'il serait possible de "traiter au moyen des mêmes principes l'analyse algébrique et l'analyse différentielle" 24 Ce qui a pu faire dire que Gomes de Souza avait pressenti des développements que l'oeuvre de Galois rendrait possible, voire en suggérant un parallèle entre Evariste Galois et Gomes de Souza, tous deux génies précoces et trop tôt fauchés ${ }^{25}$.

\section{Mathématiques et positivisme.}

Gomes de Souza représente une brillante exception dans le Brésil du dix-neuvième siècle, celle d'un physico-mathématicien qui fut peut-être de génie. Il faudra attendre les premières années du vingtième siècle pour voir apparaître à nouveau une pensée originale en mathématiques et en physique mathématique. Entretemps, ces disciplines sont enseignées dans les Ecoles d'ingénieurs en fonction de leur utilité et suivant une présentation peu propice à susciter l'innovation. De plus, cet enseignement même est menacé après l'abdication de l'Empereur et l'avènement de la République sous la bannière des idées 'positivistes', et sera d'ailleurs pendant un temps supprimé ${ }^{26}$. C'est ici que se place l'intermède positiviste'.

Si la Société pour l'étude du positivisme fut fondée à Rio par Benjamin Constant 27 , en 1870, dans l'intention de propager 1"'Apostolat positiviste", c'est à bien des années auparavant que remonte l'influence

\footnotetext{
23 Gomes de Souza [1882].

24 Ibid.

25 Freire [1931]. Gomes de Souza ne connaissait pas l'oeuvre de Galois. Freire évoque l'extension de la théorie de Galois aux équations différentielles, qui fut l'oeuvre d'Emile Picard.

26 A partir de 1896 à l'Ecole Polytechnique de Rio (Oliveira de Castro [1955], p. 62).

27 Benjamin Constant Botelho de Magalhães (1836-1891) fut adepte de la doctrine de Comte dès 1857.
} 
de la doctrine d'Auguste Comte au Brésil. Les premiers disciples brésiliens de Comte furent des ingénieurs formés à Paris - notament à l'Ecole Polytechnique comme élèves étrangers -, qui avaient suivi ses cours dès les années $1830^{28}$. L'influence du positivisme s'exerça ainsi de manière privilégiée au Brésil par le canal des enseignants des Ecoles d'ingénieurs ${ }^{29}$ : il n'y eût pas, dit-on, un ingénieur civil ou militaire qui n'aît été à cette époque enrôlé dans la doctrine ${ }^{30}$. Nul doute que le prestige de Comte auprès d'eux ne fût dû à sa qualité de mathématicien.

La première mention du positivisme au Brésil figure (par une citation de Comte) dans une thèse de dissertation sur les principes de la Statique, soutenue en 1850 à l'Ecole Militaire. C'est dans cette même Ecole, ainsi qu'à l'Ecole Polytechnique de Rio, qu'enseignèrent plus tard Benjamin Constant et ses disciples Miguel Lemos et Raimundo Teixeira Mendes: peu à peu, sous leur influence et celle d'autres professeurs également convertis à la doctrine ${ }^{31}$, l'enseignement supérieur des mathématiques et de la physique s'orienta délibérément dans la direction 'positiviste', telle que Comte la présentait dans sa Géométrie analytique et dans sa Synthèse subjective ${ }^{32}$. Dans ce dernier ouvrage, Comte rejetait les constructions abstraites des nouvelles mathématiques (représentées notamment par Cauchy) et enseignait que les mathématiques étaient achevées avec la Mécanique céleste, la seule tâche dans ce domaine étant, désormais, une systématisation finale subordonnée à l'ensemble des connaissances humaines.

Par son caractère unilatéral, le 'positivisme mathématique' (d'ailleurs prolongé dans l'oeuvre de Pierre Lafitte, auquel les positivistes brésiliens étaient liés ${ }^{33}$ ) orientait au Brésil une direction bien différente de celle des traditions-mères: les mathématiques et la physique mathématique s'arrêtaient à celles des Laplace, Monge, Fourier, Poinsot, et se poursuivaient indifférentes à toutes les

28 Lins [1964], éd. 1967, p. 13 et suiv.

29 Ceci dit sans oublier que le positivisme fut enseigné et divulgué de manière systématique dans les Ecoles de Droit - notamment pour la première fois à Recife, où l'Ecole de Droit et de Philosophie fut fondée par Tobias Barreto - et fut également très répandu dans les Ecoles de Médecine.

30 Freire [1953].

${ }^{31}$ Lins [1964], p. 289 et suiv. Les mathématiciens positivistes les plus notoires étaient, selon Luiz Freire [1953], Benjamin Constant, les frères Morais Rêgo, Samuel de Oliveira, José Eulálio, Ferreira Braga, Roberto Trompowski, etc.

32 Comte $[1843,1856]$.

33 Cf. en part. Lafitte [1875]. 
transformations qui, de Cauchy à Poincaré et Hilbert, s'opéraient au même moment en Europe, et en particulier en France même, où d'ailleurs se répandaient des manuels traitant des nouvelles conceptions et méthodes, comme le Cours d'Analyse de l'Ecole Polytechnique de Cauchy ou, plus tard, le Cours d'Analyse de Camille Jordan ${ }^{34}$.

Cet effet du positivisme d'Auguste Comte et ses disciples sur les mathématiques constitue un avatar spécifiquement brésilien : on ne constate pas de situation semblable dans l'enseignement des mathématiques en France. D'ailleurs 1'“Apostolat positiviste” brésilien reprenait un flambeau déjà bien chancelant en Europe. Il éditait et diffusait à Paris, Londres et Rio les oeuvres de Comte, ainsi que les livres d'une collection à l'usage des professeurs et des élèves des Ecoles Militaires, Navales et Polytechniques ${ }^{35}$. On trouvera un indice de la perte de vitesse du positivisme - voire d'un désintérêt pour la conception comtienne des mathématiques - dans la patrie d'origine de la doctrine à considérer les circonstances de la ré-édition de la Géométrie analytique de Comte par les soins de Miguel Lemos. Ce dernier avait déjà traduit l'ouvrage en portugais, avec Teixeira Mendes, mais se ravisa et le fit paraître dans la langue originale pour lui assurer une diffusion plus large - donc, internationale -, selon ce qu'il annonce dans une lettre à Pierre Lafitte $^{36}$.

La figure et l'oeuvre du maréchal Trompowski fournissent la meilleure illustration possible du positivisme mathématique et de son influence. Elève de Benjamin Constant à l'Ecole Militaire, puis son successeur, il publia entre 1903 et 1905 quatre épais volumes, totalisant 4000 pages, de Leçons de géométrie algébrique, de géométrie différentielle, de géométrie intégrale et d'algèbre supérieure ${ }^{37}$. Luiz

\footnotetext{
34 Comme le souligne Freire [1953], qui oppose Cauchy à Poinsot en ces termes : "deux enseignements, deux écoles, l'une qui mourait, l'autre qui surgissait avec Cauchy". Pour Freire, le positivisme au Brésil reprenait, figeait et prolongeait la résistance des conceptions anciennes aux idées nouvelles. On voit combien, dans sa critique des 'mathématiques positivistes' brésiliennes, la référence de Luiz Freire va encore préférentiellement aux mathématiciens français.

35 Cette collection fut lancée en 1893.

36 Miguel Lemos, lettre à Pierre Lafitte du 7.8.1879, in Lins [1964], éd. 1967, p. 595. Cf. Comte 1894: l'ouvrage est co-édité à Paris et à Rio.

37 Les Leçons de géométrie algébrique [Trompowski 1903], par exemple, sont dédiées à la mémoire d'Auguste Comte, et portent en exergue une citation du Maître. Elles sont marqués par un souci de classification, pour une science achevée, très comtien. Le Maréchal publia également dans la Revista Maritima et dans la Revista dos docentes militares, un autre essai systématique d'étude, par la géométrie algébrique, du plus grand
} 
Freire, qui prononça son hommage pour le centenaire de sa naisance ${ }^{38}$, voit dans cette oeuvre "le plus grand effort didactique à ce jour au Brésil en mathématiques", loue la clarté de l'exposé, et dit avoir lui-même appris "les classiques de la pensée mathématique" dans les ouvrages du maréchal, qu'il qualifie d'ailleurs de "meilleur des maîtres dans les limites de l'orientation suivie, qui était l'orientation positiviste". Mais l'éloge est mitigé, venant après une longue analyse, qui occupe plus de la moitié du discours, des méfaits du positivisme au Brésil en matière de sciences, et singulièrement en mathématiques; et la critique qui le suit n'en est que plus vive, soulignant l'ignorance et le rejet, dans les ouvrages du militaire et mathématicien positiviste, des oeuvres, qui lui étaient contemporaines, des "Cauchy, Weierstrass, Poincaré, Painlevé, Borel, Lebesgue, qui furent les Maîtres des mathématiques de son temps". En déplorant la stérilisation des esprits qui en est résultée, et qui a pu empêcher l'épanouissement de grands talents, Luiz Freire ne fait qu'exprimer le sentiment général des scientifiques des générations suivantes.

L'influence des positivistes en matière d'enseignement perdurera dans les premières décennies du vingtième siècle, et c'est en réaction contre elle que l'Université sera instituée ${ }^{39}$. Elle retarda probablement l'instauration d'une recherche originale - elle tendait à en dissuader, plutôt qu'à y inciter -, du moins en ce qui concerne les mathématiques et la physique. Mais les connaissances les plus contemporaines étaient malgré tout présentes par les livres et les revues, dans les bibliothèques mêmes des Ecoles, et ceux qui les cherchaient avaient la possibilité de les trouver. Tel fut le cas de Oto de Alencar, pourtant éduqué dans les conceptions positivistes, et qui sut se défaire de ce "moule rigide", de cette "carapace" 40.

nombre de courbes possibles. Les quatre volumes des Leçons furent les manuels utilisés au cours des vingt premières années du siècle, dans les Ecoles d'ingénieurs et les Ecoles militaires.

38 Freire [1953].

39 Cf. Paim 1977.

40 Selon les expressions de Luiz Freire [1953]. 


\section{La Physique au Brésil dans les premières années du vingtième siècle : contributions critiques et compréhension originale.}

\section{Contributions critiques en physique mathématique : la stabilisation d'une tradition.}

Oto de Alencar Silva ${ }^{41}$, mathématicien et astronome, appartint en effet un temps au courant positiviste alors dominant dans la discipline, avant de s'élever contre la rigidité et la fermeture de cette doctrine. L'article dans lequel il relevait "des erreurs de mathématiques dans la synthèse subjective d'Auguste Comte"42 causa quelque sensation au-delà même du cercle des professeurs-ingénieurs de l'Ecole Polytechnique de Rio. Formé dans cette Ecole, il y enseigna, et publia dans la Revue qui y fut fondée, dès 1897, la plupart de ses travaux. Il publia aussi plusieurs articles dans des revues de mathématiques au Portugal et en France. C'est par lui que la recherche originale mathématique et physico-mathématique fit au Brésil sa réapparition, dans la lignée de Gomes de Souza ${ }^{43}$ et en relation aux développements récents, permettant, par ses travaux originaux et surtout par son enseignement, de dépasser les limitations de l'imprégnation positiviste. Les travaux de l'école française, en particulier, trouvaient en lui un connaisseur averti. Son travail sur les surfaces minimales de Riemann, par exemple, fait référence aux considérations de Darboux et Poincaré sur le sujet, notamment aux travaux de Poincaré sur la capillarité, phénomène où l'on a affaire à de telles surfaces ${ }^{44}$.

Oto de Alencar s'intéressa à des problèmes de physique mathématique, comme ceux de l'astronomie théorique (théorie de la Lune, etc.), mais également à la théorie physique elle-même, jusque dans sa relation à l'expérience. Son article sur "la théorie de Maxwell et les oscillations hertziennes", paru en 1899, présente et analyse l'opuscule

\footnotetext{
41 Sur Oto de Alencar Silva (1874-1912), cf. Amoroso Costa [1929 (éd. 1981)], p. $67-$ 86, Costa Ribeiro [1955], p. 168-170, Morais [1955], p. 147-148, Oliveira Castro [1955], p. 65-67.

42 Alencar Silva [1898]. Les erreurs relevées ont trait à certains énoncés géométriques de Comte.

43 En particulier par un travail de 1901, publié en français dans une revue portugaise, sur la propagation du son, qui prolonge un des mémoires de Gomes de Souza (Alencar [1901]).

44 Ainsi qu'à des études parues dans les Annales de l'Ecole Normale Supérieure de Paris.
} 
publié peu d'années auparavant sous ce titre par Poincaré ${ }^{45}$. Il écrit sur tels problème d'électrostatique, sur l'effet Zeeman, propose une nouvelle démonstration de la formule de Stokes, présente, en 1906, un recueil de notes et de mémoires sur la physique et l'électrotechnique, et un autre sur la théorie des erreurs.

Les résultats réellement inédits concernent surtout quelques problèmes mathématiques (comme les applications géométriques des équations de Ricatti), et la plupart de ses mémoires, articles et notes de cours sont de caractère didactique, menées par un vif sens critique, exercices de réflexion plutôt que recherches originales. C'est là un trait général de la plupart des contributions de la génération de mathématiciens et physiciens théoriciens qui va le suivre immédiatement; on n'en sous-estimera pas le rôle formateur, ces exposés critiques s'avérant propres à aiguiser la compréhension profonde et, par là, à stimuler des pensées originales. Oto de Alencar disparut trop jeune ${ }^{46}$ pour mesurer le fruit de son enseignement, et c'est son disciple Manuel Amoroso Costa qui devait reprendre le flambeau dans la même direction. Mais nous sortons, avec ce dernier, de la période stricte à laquelle nous voulions nous cantonner.

Faute de place, nous laisserons de coté ce qui a trait à la physique expérimentale, dont les premiers linéaments furent posé par Henrique Morizé, d'origine française, et dont le rôle fut également important à la direction de l'Observatoire national et à la Chaire de physique expérimentale de l'Ecole Polytechnique de Rio ${ }^{47}$.

\footnotetext{
45 En 1894.

46 En 1912, dans la trentaine. Amoroso Costa devait également disparaître jeune, accidentellement, en 1928.

47 Cf., en part., Rocha e Silva [1976].
} 


\section{Voies et vecteurs de la communication.}

Si les scientifiques brésiliens cultivent les mathématiques avancées et la physique fondamentale, c'est, dans toute la période étudiée, à titre purement individuel. Le rôle que les métropoles ont joué dans la formation et dans la circulation des débats sur les nouvelles idées scientifiques, pour indirect qu'il aît été, n'en fut pas moins essentiel. Cette influence s'est effectuée par les livres et les revues publiés en Europe, présents pour bon nombre d'entre eux dans les bibliothèques des institutions brésiliennes, ou acquis individuellement par abonnements et commandes, notamment par l'intermédiaire des actives librairies de Rio. A quoi s'ajoute la répercussion immédiate et importante, dans la presse brésilienne, des informations scientifiques données dans les périodiques d'Europe.

Plusieurs traits caractérisent ce mode spécifique de communication et d'influence, dans ses effets sur le 'milieu' récepteur. L'un est relatif à la langue, et renforce une influence par ailleurs dominante en ce qui concerne la culture et les idées: les publications en langue française sont privilégiées, la France, outre son influence culturelle en général, étant alors celui des grands pays européens scientifiquement développés dont la langue était lue couramment par les élites sociales qui avaient accès à l'enseignement. Rappelons d'ailleurs que le commerce des livres au Brésil, au dix-neuvième siècle comme au début du vingtième, était presque un apanage de français installés au pays, et cela vaut aussi bien pour les ouvrages généraux que pour les publications scientifiques ${ }^{48}$.

D'un autre coté, que le vecteur de la communication des connaissances nouvelles fût exclusivement l'écrit imprimé et publié, y compris en ce qui concerne les débats d'idées, entraîne une différence avec ce que serait un enseignement oral et une participation directe à des discussions. Cette différence est de deux ordres: le délai d'une communication différée, et l'espèce d'autorité particulière que donne la chose écrite par les Maîtres, dans sa présentation formelle, comme un produit achevé. Telle avait été, certes, la voie naturelle de la communication dans le passé: mais elle n'était plus la seule désormais, les conditions de la production scientifique ayant changé. Etant donné ce qu'est désormais le système des connaissances, cette voie, tout en rendant la communication possible, ne facilite pas la participation: elle

48 Par ex., les librairies Garnier, Briguiet, etc., à Rio, Garraux à São Paulo. Cf. Hallewell 1985. 
favorise l'assimilation plutôt que la création.

De fait, les contributions des physico-mathématiciens brésiliens, jusqu'aux années trente de ce siècle - sauf exceptions marquantes -, ne constituent pas tant des recherches originales que des commentaires et présentations critiques des idées scientifiques nouvelles en provenance des métropoles. Ce qui ne préjuge pas de la qualité de ces contributions, souvent très pertinentes et manifestant une pénétration profonde des problèmes, révélatrices de la qualité exceptionnelle de professeurs qui auraient de toute évidence pu, dans un contexte favorable, réaliser des recherches de premier plan. Mais, d'un autre coté, le caractère différé de la participation aux connaissances nouvelles et de leur assimilation favorise sans doute la prise de recul, lisible dans l'intérêt marqué pour l'aspect philosophique des problèmes. Ce dernier est d'ailleurs cultivé, dans toute cette période, par la fréquentation d'ouvrages de philosophie des sciences comme ceux de Poincaré ou d'autres savants et philosophes, notamment les auteurs de la Bibliothèque de philosophie contemporaine, dont les titres furent largement diffusés voire réimprimés par les libraires ${ }^{49}$.

\section{Références.}

- Alencar Silva, Oto de 1898. "Alguns erros de mathemática na Synthese subjectiva de A. Comte", Revista da Escola Polytechnica (Rio de Janeiro) 2, 1898 (n9-10), 113-130. - Alencar Silva, Oto de 1901. "De l'action d'une force accélératrice sur la propagation du son", Jornal de Ciências Matématicas e Astronômicas (Porto), 1901.

- Amoroso Costa, Manoel 1929. As idéias fundamentais da matemática, Pimenta de Mello, Rio de Janeiro, 1929. 3a ed. aum., Convivio/EDUSP, São Paulo, 1981, p. 169330 .

- Arantes, Paulo Eduardo 1988. "O positivismo no Brasil", Novos Estudos (CEBRAP, São Paulo), n 21, junho de 1988, 185-194.

- Arbousse-Bastide, Paul 1957. Les disciples brésiliens d'Auguste Comte, Deuxième thèse (non publiée), Paris, 1957.

- Azevedo, Fernando de 1943. A cultura brasileira, 3 vols., Melhoramentos, São Paulo, 1947. 3a ed., 1958.

- Azevedo, Fernando de (dir.) 1955. As ciências no Brasil, 2 vols., Melhoramentos, São Paulo, 1955.

- Ben-David, Joseph (1970). "The rise and decline of France as a scientific center", Minerva, $\mathrm{n}^{\circ}$ 8, 1970, 160 et suiv. Repris dans Ben-David, J., The scientist's role in

49 On en trouve aujourd'hui encore de nombreux exemplaires dans les boutiques de livres d'occasion. 
society. A comparative study, Prentice Hall, 1971.

- Boltzmann, Ludwig (1905). "Reises eines deutschen Professors ins Eldorado", in Boltzmann, L. Populäre Schriften, Barth, Leipzig, 1905. Trad. fr. par Ulrike Bockskopf et Didier Aviat, Voyage d'un professeur allemand en Eldorado et autres écrits populaires, Actes Sud, Arles, 1987.

- Coggiola, Osvaldo (org.) 1990. A Revolução francesa e seu impacto na América latina, Nova Stella/CNPq/EDUSP, São Paulo, 1990.

- Comte, Auguste 1843. Traité élémentaire de géométrie analytique à deux et à trois dimensions, Carilian-Goeury et Dalmont, Paris, 1843. Reproduit in Comte 1894.

- Comte, Auguste 1856. Synthèse subjective ou système universel des conceptions propres à l'état normal de l'humanité, 1856. t.1 : Traité de philosophie mathématique, Chez l'auteur, Paris, 1856.

- Comte, Auguste 1894. La géométrie analytique d'Auguste Comte, nouvelle édition précédée de La Géométrie de Descartes, Bahl, Paris / Briguiet, Rio de Janeiro, 1894.

- Costa Ribeiro, J. 1955. " A física no Brasil", in Azevedo [1955], vol. 1, p. 163-202.

- Dantes, Maria Amelia M. 1987. "A presença francesa e a formação de tradições em ciências exatas e naturais no Brasil", in Table-ronde Images réciproques Brésil-France, Paris, 1987. (A paraître.)

- Ferri, Mario G. e Motoyama, Shozo 1979-1981. História das ciências no Brasil, 3 vols., EDUSP/CNPq, São Paulo, 1979-1981.

- Freire, Luiz, 1931. "Joaquim Gomes de Souza", Revista Brasileira de Matemática, ano $3,1931, \mathrm{n}^{\circ} 1$ (setembro), 1-8.

- Freire, Luiz 1953. [Discours d'hommage au Maréchal Trompowsky], 177a Sessão do Conselho Delberativo do Conselho Nacional de Pesquisa, realizada a 27 de Outubro de 1953, em conjunto com a Academia Brasileira de Ciências (...) em comemoração do centenario de nascimento do Marechal Roberto Trompowsky Leitão de Almeida (mimeogr., Archives Luiz Freire).

- Gilpin, Robert 1968. France in the age of the scientific state, Princeton University Press, Princeton (N.J), 1968.

- Gomes de Souza, Joaquim 1848. Dissertação sobre o modo de indagar novos astros sem o auxilio de observações diretas, Tip. Teixeira, Rio de janeiro, 1848.

- Gomes de Souza, Joaquim 1882. Mélanges de Calcul Intégral, Préface de Charles Henry, Brockhaus, Leipzig, 1882.

- Hallewell, Laurence 1985. O livro no Brasil (sua historia), T.A. Queiroz/EDUSP, São Paulo, 1985.

- Lafitte, Pierre 1875. Calcul arithmétique. Cours professé par M. Pierre Lafitte suivant le plan tracé par Auguste Comte dans sa Synthèse Subjective (Année 1875). Leçons rédigées par M. Ch. Jeannolle. Enseignement positiviste, Rio de Janeiro, 1880.

- Lins, Ivan 1964. História do positivismo no Brasil, Comp. ed. nacional, São Paulo; 2 è éd., 1967.

- Mathias, Simão 1982. "Evolução da pesquisa cientifica no Brasil", Interciencia 7, 1982 (n 6, nov.-dec.), 340-343.

- Morais, Abraão de 1955. "A astronomia no Brasil", in Azevedo [1955], vol. 1, p. 81161.

- Oliveira Castro, F.M. de 1955. "A matemática no Brasil", in Azevedo [1955], vol. 1, p. 41-77.

- Paim, Antonio 1977. A Ciência na Universidade do Rio de Janeiro, Sociedade de Instrução, Instituto Universitario de Pesquisas do Rio de Janeiro, IUEPRJ, 1977, miméogr., 161 p. (Archives de l'Academia Brasileira de Ciências, Rio). 
- Paty, Michel et Petitjean, Patrick 1985. Sur l'influence scientifique française au Brésil entre le dix-neuvième et le vingtième siècles, Cahiers des Amériques Latines, nlle série, $\mathrm{n}^{\circ} 4$, hiver 1985, 31-48.

- Paty, Michel 1987. "Exemple d'influence scientifique et culturelle: les voies spécifiques de la physique mathématique", in Table-ronde Images réciproques BrésilFrance, Paris, 1987. (A paraître.)

- Paty, Michel (en prép. 1). "De la physique mathématique à la physique théorique (Brésil, les années 20 et 30): influences et constitution d'une voie originale", in Dantes, Maria Amelia ; Hamburger, Amelia ; Paty, Michel et Petitjean, Patrick (eds.), Estudos historicos sobre colaboração cientifica e tecnologica e as relações Brasil-França. (en préparation).

- Paty, Michel (en prép. 2). "La réception de la théorie de la Relativité au Brésil" (en préparation).

- Petitjean, Patrick 1988. "Le Groupement des Universités et Grandes Ecoles de France pour les relations avec l'Amérique latine et la créations d'Instituts à Rio de Janeiro, São Paulo et Buenos-Aires", in d'Ambrosio, Ubiratão (coord.), Anais do Secundo Congresso Latino-Americano de Historia da Ciência e da Tecnologia, São Paulo, 1988, Nova Stella/EDUSP, São Paulo, 1989, p. 443-448.

- Petitjean, Patrick 1989. "Images de la Révolution française et constitutions de traditions scientifiques au Brésil", in Vovelle, Michel (coord.), L'Image de la Révolution française. (Actes du Congrès mondial pour le Bicentenaire de la Révolutiion française, Paris, Juillet 1989), Pergamon Press, Oxford, 1989, p. 1385-1394.

- Petitjean, Patrick 1991. "Autour de la Mission française pour la création de l'Université de São Paulo", in Petitjean, Patrick, Jami, Catherine et Moulin, Anne-Marie (eds.), Sciences and Empires, Reidel, Dordrecht, 1991, ce volume.

- Ramos, Teodoro A. 1929 b. "Gomes de Souza", Conferencia realizada em 26.6.1929 na Academia Brasileira de Ciências, in Ramos, T., Estudos (Ensino, Sciencias physicas e mathematicas), Escolas profissionais do Liceu Coração de Jesus, Rio de Janeiro, 1933, p. 7-14.

- Rocha e Silva, Mauricio 1976. "Birth and development of experimental science in Brazil", Interciência I, 4, 1976.

- Schwartzmann, Simon 1979. Formação da comunidade científica no Brasil, Nacional/FINEP, São Paulo, Rio de Janeiro, 1979.

- Trompowski Leitão de Almeida, Carlos Roberto 1903. Lições de Geometria algebrica, Imprensa Nacional, Rio de Janeiro, 1903. 